\title{
Collective charge excitations along cell membranes
}

\author{
Efstratios Manousakis \\ Department of Physics, Florida State University, Tallahassee, FL 32306-4350, and \\ Department of Physics, University of Athens, Greece.
}

(Dated: October 30, 2018)

\begin{abstract}
A significant part of the thin layers of counter-ions adjacent to the exterior and interior surfaces of a cell membrane form quasi-two-dimensional (2D) layers of mobile charge. Collective charge density oscillations, known as plasmon modes, in these $2 \mathrm{D}$ charged systems of counter-ions are predicted in the present paper. This is based on a calculation of the self-consistent response of this system to a fast electric field fluctuation. The possibility that the membrane channels might be using these excitations to carry out fast communication is suggested and experiments are proposed to reveal the existence of such excitations.
\end{abstract}

PACS numbers: 87.16.Dg,87.14.Cc,82.39.Wj,82.45.Mp

Collective charge excitations in condensed matter, the so-called plasmon modes, play a fundamental role in understanding the properties of a metal ${ }^{\underline{1}}$. These are excitations of the electron gas as a whole and are responsible for the electromagnetic response of the metal for frequencies above and below the classical plasma oscillation frequency, and for our understanding of other effects such as screening. The plasmon is the quantum of such collective charge oscillations which may exist in any charged system and in fact they were first discovered in a classical plasma 2 . In quasi-two-dimensional systems such as surfaces of metals $3.4,5.6 .7 .8$, or interfaces of metals with insulators, plasmons have been investigated both theoretically ${ }^{5.6 .7 .8}$ and experimentally ${ }^{3.4}$ for several decades.

Plasmon modes have been found ${ }^{9}$ in a system of electrons on the surface of liquid helium. The surface of a lipid bilayer with the surrounding counter-ion system, which we consider in this paper, is analogous to that of electrons on helium surface as will be made clear below. The surface of a cell membrane in aqueous environment becomes negatively charged ${ }^{15}$. It is wellknown 10.11.12.13.14 that a diffuse double layer of counterions from the solution screens the negative charge, thus, a thin two-dimensional (2D) layer of mobile cations is accumulated adjacent to the extra-cellular and intra-cellular membrane surfaces. In addition, because of different permeabilities of the cell membrane channels to various cations, the inside of the cell is kept at a negative potential relative to the outside. In this paper it is pointed out that the thin layers of counter-ions adjacent to the lipid bilayer are characterized by 2D longitudinal plasmon excitations. These modes are excited in response to a fast perturbation such as the opening of an ion channel. The gating mechanism of ion channels along the cell membrane, that are believed to open using voltage sensing gates, is an open problem in cell biology ${ }^{15.16}$. The collective charge density wave which is theoretically found in this paper can play the role of a very fast signal carrier for such ion channel communication. Therefore the collective modes investigated here can play a fundamental role in cell biology.
The negatively charged exterior and interior surfaces of biological membranes of cells $\frac{17}{17}$ as shown in Fig. 1 where the density of charged phospholipid heads is higher on the exterior membrane surface. Diffuse double layers of counter-ions, such as $\mathrm{H}^{+}, \mathrm{Na}^{+}, \mathrm{K}^{+}, \mathrm{Ca}^{++}, \ldots$, form on each membrane side. Due to the different membrane permeability for these cations, there is a voltage $-V$ (of the order of $-100 \mathrm{mV}$ ) between the cell interior relative to the exterior. The solid line in Fig. 1 is the positive free counter-ion distribution near the membrane surfaces 17 . In thermal equilibrium the counterion charge density varies as we move away from the membrane surface as shown, within the so-called GouyChapman(GC) length $\lambda_{G C}^{i}=k_{B} T \epsilon_{i} / 2 \pi \sigma_{i} q_{i}$ for each membrane side. Here $\epsilon_{i}$ and $q_{i}$ are the dielectric constants and the counter-ion charges of the outside and inside of the cell. The surface densities $\sigma_{i}=q_{i} n_{i}^{0}$ of both membrane sides are experimentally accessible and they can be as high as $n^{0} \sim 10^{-2} \AA^{-2}$ or even higher. This counter-ion surface charge distribution is controlled by the bulk cation concentrations of the intra-cellular and extra-cellular fluids ${ }^{13}$. In addition, depending on the temperature, cation concentration, and cation charge, a fraction of these ions can be adsorbed on the membrane surface. The adsorbed ion density versus bulk aqueous ion concentration is determined from the Stern adsorption isotherm theory $\underline{14}$ and its extensions ${ }^{18,19}$. The approximate value of $\lambda_{G C}$ is about $4 \AA$, if we use $T=300 \mathrm{~K}$, $\epsilon=80$ and $n^{0}=10^{-2} \AA^{-2}$. For charge fluctuations propagating with wave vectors $\mathbf{k}$ parallel to the surfaces and magnitude $k<<1 / \lambda_{G C}$ (which is the case of our interest), these layers can be regarded as two-dimensional.

In this paper, the collective charge response of the mobile counter-ion charge of the interior and exterior surface layers to an external electric field perturbation is studied. It is shown that there are longitudinal charge collective modes with wave vectors parallel to the surface and the two layers are coupled and fluctuate together as a whole in the limit where the wavelength is much longer than the membrane thickness $d$.

The counter-ions of the diffuse double layer adjacent to the extra-cellular membrane surface are character- 
ized by mass $m_{1}$ charge $q_{1}=Z_{1} e$ ( $Z_{1}$ the ionic valence and $e$ the electron charge) and instantaneous surface number density $n_{1}(\mathbf{r}, t)(\mathbf{r}=(x, y))$ and those adjacent to the intra-cellular membrane surface by mass $m_{2}$, charge $q_{2}=Z_{2} e$ and instantaneous surface number density $n_{2}(\mathbf{r}, t)$. We are interested in studying deviations $\delta n_{i}(\mathbf{r}, t)$ from the equilibrium surface density $n_{i}^{0}$, where $\delta n_{i}(\mathbf{r}, t)=n_{i}(\mathbf{r}, t)-n_{i}^{0}$. Following the well-known approach $^{1}$ to this class of problems where there is the possibility of collective response of the charge, a perturbation $\phi_{\text {ext }}(\mathbf{r}, t)$, due to an external charge density fluctuation $\rho_{\text {ext }}(\mathbf{r}, z, t)$ and current fluctuations $\mathbf{J}_{\text {ext }}(\mathbf{r}, z, t)$, is introduced. These physical quantities are related as follows

$$
\begin{array}{r}
\nabla^{2} \phi_{\text {ext }}(\mathbf{r}, z, t)=-4 \pi \rho_{e x t}(\mathbf{r}, z, t) \\
\partial_{t} \rho_{\text {ext }}(\mathbf{r}, z, t)+\nabla \cdot \mathbf{J}_{\text {ext }}(\mathbf{r}, z, t)=0,
\end{array}
$$

where the $z$ direction is taken perpendicular to the membrane surface. This perturbation may be caused by the opening of a sodium or potassium membrane channel or by a real external probe. While these channels are part of the cell membrane, they are not part of the degrees of freedom considered here, namely we only consider the system of the accumulated charge on both sides of the cell membrane.

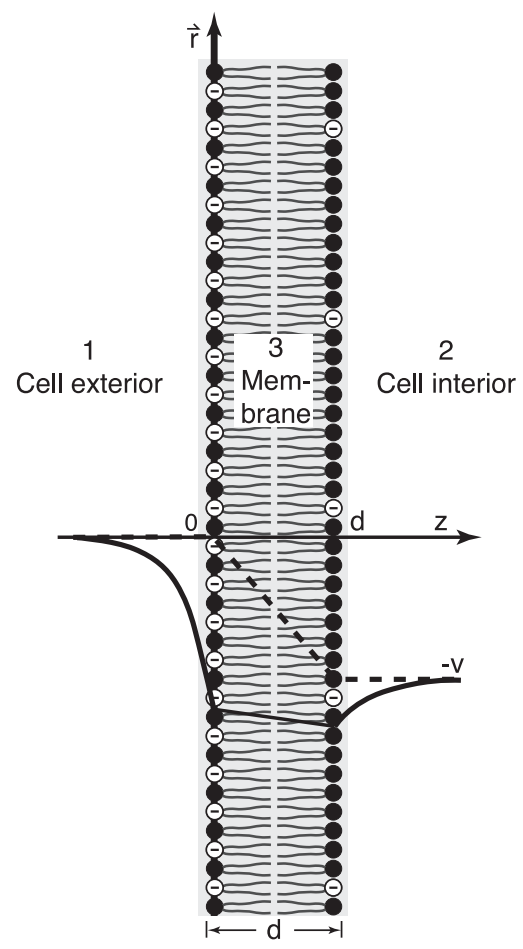

FIG. 1: The lipid bilayer cell membrane of width $\mathrm{d}$ and its various features are explained in the text.

We would like to investigate the response of the system to such an external perturbation namely to solve the
Poisson equation:

$$
\nabla^{2} \phi_{i}(\mathbf{r}, z, t)=-\frac{4 \pi}{\epsilon_{i}} \rho(\mathbf{r}, z, t),
$$

where $i=1,2$ and denotes the cell exterior and interior respectively. Here $\phi_{i}(\mathbf{r}, z, t)$ is the total field in the regions $i=1,2$ and the total charge density is given by

$$
\rho(\mathbf{r}, z, t)=\rho_{0}(z)+\rho_{e x t}(\mathbf{r}, z, t)+\rho_{\text {ind }}(\mathbf{r}, z, t)
$$

where $\rho_{0}(z)$ is the equilibrium charge density and $\rho_{\text {ind }}(\mathbf{r}, z, t)$ is the induced charge density. Within our $2 \mathrm{D}$ approximation, the induced density is written as

$$
\rho_{\text {ind }}(\mathbf{r}, t)=q_{1} \delta n_{1}(\mathbf{r}, t) \delta(z)+q_{2} \delta n_{2}(\mathbf{r}, t) \delta(z+d) .
$$

For both layers we require the validity of the linearized continuity equation

$$
\partial_{t} \delta n_{i}(\mathbf{r}, t)+n_{i}^{0} \nabla \cdot \mathbf{v}_{i}(\mathbf{r}, t)=0, \quad i=1,2
$$

where $\mathbf{v}_{i}(\mathbf{r}, t)$ is the velocity of the counter-ions along the $i^{t h}$ layer. The equation of motion for a single counter-ion maybe written as follows:

$$
\begin{aligned}
m_{i}\left(\partial_{t} \mathbf{v}_{i}+\frac{\mathbf{v}_{i}}{\tau}\right)= & -q_{i} \nabla \phi_{i}\left(\mathbf{r}, z=z_{i}, t\right) \\
& -\frac{m_{i} s_{i}^{2}}{n_{i}^{0}} \nabla \delta n_{i}(\mathbf{r}, t)
\end{aligned}
$$

where $z_{i}=0^{-}, d^{+}$for the exterior and interior layer respectively and $\tau$ is the time between collisions, while $s_{i}=\sqrt{\gamma k_{B} T / m_{i}}$ is the velocity of density fluctuations in the charged subsystems and the value of $\gamma=2$ will be used for a 2D ideal gas. The first term is the force on the counter-ion due to the local electric field while the second term is the force due to the local compression of the counter-ion 2D gas. In order to simultaneously solve Eqs. (36 7), we consider the Fourier transform (denoted by a tilde) of all the functions of the $2 \mathrm{D}$ position vector $\mathbf{r}$ and time $t$. Eq. (3) takes the form:

$$
\left(\partial_{z}^{2}-k^{2}\right) \tilde{\phi}_{i}(\mathbf{k}, z, \omega)=-\frac{4 \pi}{\epsilon_{i}} \tilde{\rho}(\mathbf{k}, z, \omega)
$$

where $\mathbf{k}$ is the wave vector parallel to the membrane surface and $\omega$ is the frequency. Eq. (8) has the following solution:

$$
\begin{aligned}
\tilde{\phi}_{i}(\mathbf{k}, z, \omega) & =\frac{2 \pi}{\epsilon_{i} k}\left[q_{1} \delta \tilde{n}_{1}(\mathbf{k}, \omega) e^{-k|z|}+q_{2} \delta \tilde{n}_{2}(\mathbf{k}, \omega) e^{-k|z-d|}\right. \\
& \left.+\int_{-\infty}^{\infty} d z^{\prime} \tilde{\rho}^{\prime}\left(z^{\prime}\right) e^{-k\left|z-z^{\prime}\right|}\right]
\end{aligned}
$$

where $\tilde{\rho}^{\prime}(z)=\rho_{0}(z)+\tilde{\rho}_{\text {ext }}(\mathbf{k}, z, \omega)$. Substituting (9) in the Fourier transform of Eqs. (67) and by eliminating the velocity variables we find

$$
\begin{aligned}
{\left[\omega\left(\omega-\frac{i}{\tau}\right)\right.} & \left.-\omega_{i}^{2}(k)\right] \delta \tilde{n}_{i}(\mathbf{k}, \omega)-\beta_{i}(k) \delta \tilde{n}_{j}(\mathbf{k}, \omega) \\
& =\gamma_{i}(\mathbf{k}, \omega)
\end{aligned}
$$


where $i, j=1,2$ or 2,1 . We have used the definitions

$$
\begin{aligned}
\gamma_{i}(\mathbf{k}, \omega) & =\frac{Z_{i}}{e} \bar{\omega}_{i}^{2} \int d z^{\prime} \tilde{\rho}^{\prime}\left(z^{\prime}\right) e^{-k\left|z^{\prime}-z_{i}\right|} \\
\beta_{i}(\mathbf{k}) & =Z_{1} Z_{2} \bar{\omega}_{i}^{2} e^{-k d} \\
\omega_{i}^{2}(k) & =Z_{i}^{2} \bar{\omega}_{i}^{2}+s_{i}^{2} k^{2} \\
\bar{\omega}_{i}^{2} & =\frac{2 \pi e^{2} n_{i}^{0} k}{m_{i} \epsilon_{i}} .
\end{aligned}
$$

The system of Eqs. (10) has a solution for the responses $\delta \tilde{n}_{i}(\mathbf{k}, \omega)$ which attain their maximum values for the following frequencies:

$$
\begin{aligned}
\Omega_{ \pm}(k) & =\sqrt{\omega_{ \pm}^{2}(k)+\left(\frac{1}{2 \tau}\right)^{2}}+\frac{i}{2 \tau} \\
\omega_{ \pm}^{2}(k) & =\frac{\omega_{1}^{2}(k)+\omega_{2}^{2}(k)}{2} \\
& \pm \sqrt{\left(\frac{\omega_{1}^{2}(k)-\omega_{2}^{2}(k)}{2}\right)^{2}+\beta_{1}(k) \beta_{2}(k)} .
\end{aligned}
$$

The frequencies $\omega_{i}(k)$ are the frequencies of plasmon oscillations of each layer when their mutual coupling (terms $\left.\beta_{i}(k)\right)$ is small, i.e., when $k d>>1$.

The frequencies are shown in Fig. 2 for the case where all the parameters of both layers are taken to be the same. The frequencies are given in units of

$$
\omega_{0}=Z \sqrt{\frac{2 \pi e^{2} n^{0}}{m d \epsilon}}
$$

and the wave-number in units of $1 / d$. In terms of these variables the frequency of each independent layer can be expressed as

$$
\begin{array}{r}
\frac{\omega^{2}(k)}{\omega_{0}^{2}}=k d\left(1+k d_{0}\right) \\
d_{0} \equiv k_{B} T \epsilon /\left(2 \pi^{2} e^{2} n^{0}\right) \simeq 5 \AA
\end{array}
$$

and the second term has a small contribution for the range of $k$ in the graph because $d \simeq 30 \AA$. The frequencies of each independent layer are shown by the middle curve. The lower frequency corresponds to in-phase charge oscillations of the two layers and the higher frequency to out-of-phase charge oscillations. It is straightforward to show that in the long-wavelength limit $(k d<<1)$

$$
\omega_{+}(k \rightarrow 0)=\sqrt{2 s_{0}^{2} k / d+\left(s_{1}^{2}-s_{0}^{2}\right) k^{2}},
$$

and in the particular case where the parameters characterizing the layers are equal, we have $c_{p}=\sqrt{s_{0}^{2}+s_{1}^{2}}$ with $s_{0}=\omega_{0} d$. Taking realistic values of the parameters we find that for $\mathrm{H}^{+}$counter-ions the frequency-scale is $\omega_{0} / 2 \pi \sim 10^{11} \mathrm{~Hz}$ and the speed is $c_{p} \sim 3000 \mathrm{~m} / \mathrm{s}$. For heavier counter-ions these scales will be smaller as both scale as $1 / \sqrt{m}$.

The group and phase velocity of the higher frequency mode and of the independent layer oscillations in the

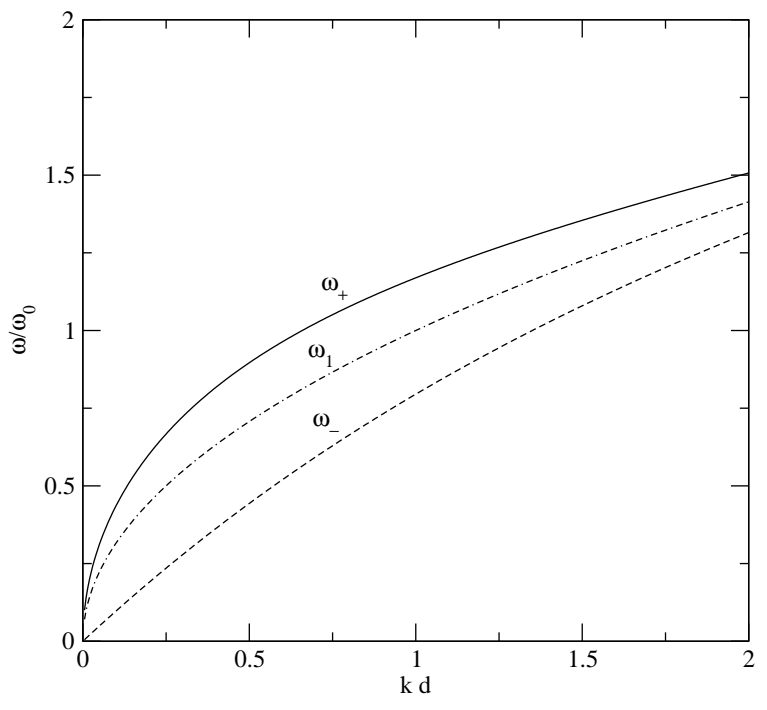

FIG. 2: Eigen-frequencies of the membrane collective charge oscillations when all characteristic parameters of the carriers of both layers are the same. The curve in the middle denoted by $\omega_{1}$ is the plasmon frequency of each layer in the limit where the coupling between the inner and outer layers is neglected. The frequencies denoted by $\omega_{-}\left(\omega_{+}\right)$correspond to in-phase (out-of-phase) oscillations of both layers together. When the parameters of the two layers are different, the corresponding plot looks similar, where instead of having one $\omega_{1}$ curve in the middle we have two, one for $\omega_{1}$ and a second for $\omega_{2}$.

long-wavelength limit diverge as $u(k \rightarrow 0)=s_{0} / \sqrt{k d}$. Therefore in the long-wavelength limit these velocities are higher than the counter-ion thermal velocities and therefore the collision frequency is lower than the collective mode frequency. Therefore the Landau damping rate is small in the long-wavelength limit. In order to obtain a more accurate expression of the plasmon frequencies instead of the bare mass and surface density, one should use the effective mass of the hydrated ion and the effective nearly-free counter-ion charge density. If there are binding sites on the membrane surface this will reduce the effective free surface charge density. Therefore more realistically we should expect frequencies below the above mentioned values. In addition, the surface corrugations of the electrostatic interaction of the counter-ions with the phospholipid surface introduce a non-zero frequency gap $\Omega_{0}$ at zero plasmon momentum. This $\Omega_{0}$ is the frequency of local charge oscillation near a local minimum of the interaction potential between the counter-ion and the membrane surface. When the surface charge density is low, this interaction may lead to the formation of a Wigner crystal where the counter-ions will order in a triangular or other lattice structures 20 . These structures of ordered charged layers have been observed in electronic bilayer systems, consisting of two quasi-two-dimensional layers of electron or hole liquids 21 . Such a Wigner crystal will be characterized by plasmon modes with frequency given by the same form as Eqs. (15/16) with the masses 


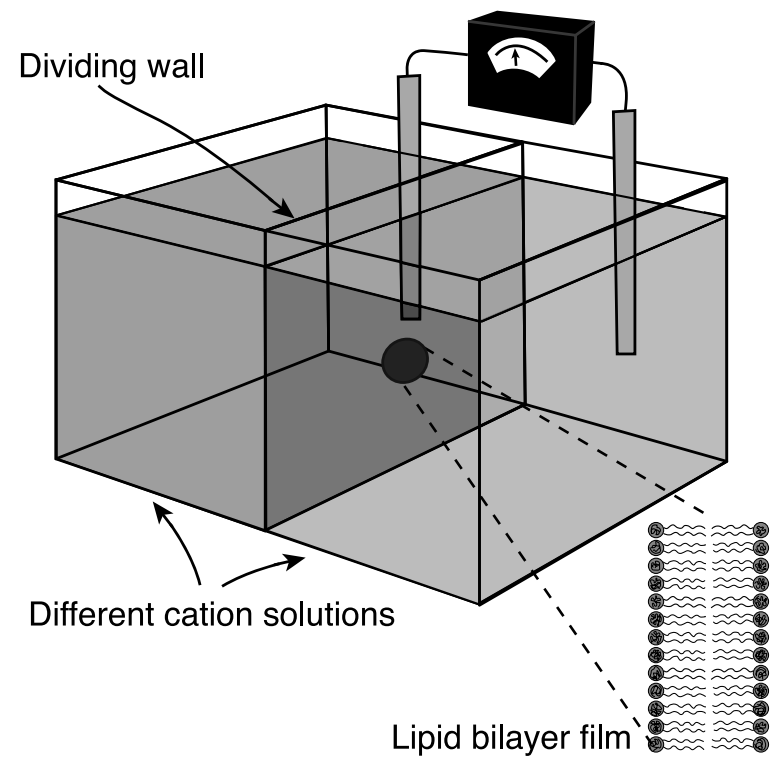

FIG. 3: An idealized experimental set up to control the cation concentration on both membrane sides.

replaced by the ion effective mass within the Wigner crystal.

These collective modes can be investigated experimentally by studying the electromagnetic absorption spectrum in the frequency region of $10^{10}-10^{11} \mathrm{~Hz}$ (depending on the wavelength of the excited plasmon and the counter-ion parameters and distribution) of artificial phospholipid bilayer membranes which separate aqueous solutions of different ionic concentrations (see Fig. 3) with high counter-ion concentrations $\frac{15}{5} \sigma_{i}$ near the ex- terior and interior surfaces. Up to $10 \mathrm{~mm}^{2}$ size membrane can be formed in the hole of the wall separating the two aqueous compartments shown in Fig. 3 by appropriately mixing the phospholipids as was first discovered by Mueller et al. ${ }^{22}$. An appropriate voltage can be applied between the two aqueous compartments of different cation solutions separated by the membrane. Plasmon modes of the two thin counter-ion layers adjacent to the membrane can be excited and probed by a source/spectrometer system. With the experimental techniques and source/spectrometer systems available today, it is possible to study the AC electromagnetic response of very small samples over a range of frequencies and to cover the above frequency domain with one or a combination of source/spectrometer systems. The plasmon frequencies should scale as $\sqrt{\sigma}$ with respect to surface concentrations (which can be estimated from the bulk concentrations) and as $1 / \sqrt{m_{i}^{*}}$ with respect to various counter-ion effective masses.

These 2D plasmon oscillations can be used by the various parts of the cell membrane to carry out communication. For example, these modes are excited in response to a cell membrane pore opening which creates a local charge distribution fluctuation. The phenomenological studies of the behavior of the gates of the voltagesensitive ion channels in cell membranes indicate a high sensitivity 15 of the voltage sensor to small changes of the membrane electric field. This implies that the 2D plasma oscillations could play a role in triggering the sensor to open the gate. Before, however, one proceeds further to study such possibilities it is important to carry out an experimental study to verify the existence and the properties of these plasmon modes.
1 D. Bohm and D. Pines. Phys. Rev. 92, 609 (1953). D. Pines Rev. Mod. Phys. 28, 184-198 (1956).

2 L. Tonks, and I. Langmuir. Phys. Rev. 33, 195 (1929).

${ }^{3}$ H. Raether. Surface excitation on smooth and rough surfaces and on gratings. Springer tracts in modern physics, V 111, Springer-Verlag (1988).

4 T. H. Geballe, Scientific American, 225, 22 (1971).

${ }^{5}$ R. H. Ritchie. Phys. Rev. 106, 874-881 (1957).

${ }^{6}$ R. A. Ferrell. Phys. Rev. 111, 1214-1222 (1958).

7 F. Stern, Phys. Rev. Lett. 18, 546 (1967).

8 A. L. Fetter Ann. Phys. 81, 367-393 (1973).

9 C. C. Grimes and G. Adams. Phys. Rev. Lett., 36, 145-147 (1976).

10 G. Gouy, J. Phys. Radium (series 4) 9, 457 (1910).

11 D. L. Chapman, Phil. Mag. (series 6), 25, 475 (1913).

12 P. Debye and E. Hückel, Phys. Z. 24, 305 (1923). and Phys. Z, 25, 145 (1924).
13 D. C. Grahame, Chem. Rev. 41, 441 (1947).

14 O. Stern, Z. Elektrochem. 30, 508 (1924).

15 B. Hille. Ionic channels of excitable membranes. Sunderland, Mass, Sinauer Associates Inc. (2001).

16 C. A. Ahern and R. Horn, Trends Neurosci, 27, 303 (2004).

17 B. Hille, A. M. Woodhull and B. J. Shapiro. Phil. Trans. R. Soc. Lond. B 270, 3 01-318 (1975).

18 J. A. Cohen and M. Cohen, Biophys. J. 36, 1981 (1981).

19 S. A. Tatulian, J. Colloid Interface Sci. 175, 131 (1995).

${ }^{20}$ V. I. Valtchinov, G. Kalman, and K. B. Blagoev, Phys. Rev. E, 56, 4351 (1997).

21 T. B. Mitchell, J. J. Bollinger, D. H. E. Dubin, X.-P. Huang, W. M. Itano, R. H. Baughman, Science, 282, 1290 (1998).

22 P. Mueller, D. O. Rudin, H. Ti Tien and W. C. Wescott, Nature, 194, 979 (1962). 